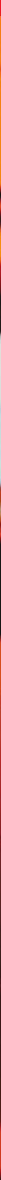

\title{
Breaking the billion-hertz barrier
}

\section{Researchers in France have switched on the world's most powerful nuclear magnetic resonance instrument. Ananyo Bhattacharya asks whether it will attract new life to NMR spectroscopy.}

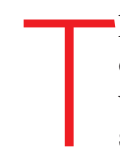

he 12-tonne, 4.5-metre-tall machine does little to betray the fact that it is working. No flashing lights break its smooth, white cylindrical surface. It makes no noise, not even a hum. But an incautious step over the yellow-and-black striped tape on the floor 12 metres from the world's most powerful nuclear magnetic resonance (NMR) spectrometer will erase the data on one's credit cards. Individuals with pacemakers are warned to go no closer.

It is the magnet that makes this spectrometer (pictured) at the European Centre for High Field NMR (CRMN) in Lyon, France, unique and hugely powerful. In a magnetic field, atomic nuclei with an odd number of protons or neutrons will resonate when pulsed with the right radio-wave frequency. The frequency at which they resonate depends on the chemical environment of the atom - the type of atoms that surround it, and their proximity. By gauging the resonance frequencies for a compound, researchers can map the relative positions of atoms and the structure and identity of the molecule. It is a technique that researchers have used to great effect since the first commercial 30-megahertz (MHz) machines became available in the 1950s.
The more powerful the magnet, the more spectroscopists can 'see' - picking up tiny traces of chemicals in complex mixtures of body fluids or environmental samples, for example. The magnet at the core of the Lyon spectrometer can generate a massive 23.5-tesla field. But they give it the designation 1 gigahertz $(\mathrm{GHz})$ - the resonance frequency of a single hydrogen-1 nucleus in a field of this strength. As the most powerful of its kind, the machine represents a milestone.

Lyndon Emsley, the CRMN's scientific director who worked for seven years with the federal and regional governments and the CNRS, France's basic-research agency, to buy the instrument for $€ 11.7$-million (US\$16.3million), says that the machine will attract Europe's best chemists and structural biologists. A huge swathe of science is set to benefit from the added field strength, he says, which will reveal structures of bigger, more difficult to analyse proteins, and enable a clearer look into complex mixtures of chemicals and metabolites. But the machine has some work to do to prove that this milestone is more than symbolic. An increase of $50 \mathrm{MHz}$ over other high-field machines hardly matches the vast improvements in power made during NMR spectroscopy's heyday in the 1970s and 1980s, when superconducting magnet coils vastly enhanced field strength. Even Emsley's father, a chemist at the University of Southamphton, UK, who had long used NMR spectroscopy for his studies of liquid crystals, calls the step to $1 \mathrm{GHz}$ "incremental". Nevertheless, Emsley the younger predicts that the behemoth will prove its mettle.

One reason for his optimism is the way in which biologists, particularly structural biologists, have produced demand for increasingly powerful machines. Emsley suspects that as much as half the time on the new machine could go to cracking the atomic structures of proteins.

\section{Protein power}

NMR spectroscopy provides advantages over its main rival, X-ray crystallography. Crystallography can resolve protein structures quickly - sometimes in hours or days, compared with months for NMR spectroscopy - but only after crystals of the protein have been grown, a trial-and-error process that can add months or years to a project. In addition, NMR spectroscopy can yield useful information about proteins without a well-defined structure, including those that are extremely difficult to 
crystallize because they assume a shape only when they bind to another protein.

Charles Sanders, a biochemist at Vanderbilt University School of Medicine in Nashville, Tennessee, and his collaborators recently used an $800-\mathrm{MHz}$ machine to determine the structure of diacylglycerol kinase, one of the largest membrane proteins solved by NMR spectroscopy to date (W. D. Van Horn et al. Science $324,1726-1729 ; 2009)$. But he says that the $1-\mathrm{GHz}$ machine will appeal to those working on large proteins and membrane-bound proteins, which are extremely difficult to crystallize. For the latter, NMR-spectroscopy researchers can use lipid constructs called bicelles, which mimic cell membranes allowing bound proteins to assume a more natural shape. Some are even beginning to use nanodiscs, which corral lipid and protein together, maintaining them in their natural conformation (see image, right).

A technique developed in the late 1990s called transverse relaxation optimized spectroscopy (TROSY), has allowed NMR spectroscopy to deduce the structures of ever larger molecules by labelling the protein with different isotopes (K. Pervushin et al. Proc. Natl Acad. Sci. USA 94, 12366-12371; 1997). The physical properties that TROSY exploits are predicted to give the sharpest possible peaks at fields of up to about 1.2 GHz, so the new machine could allow spectroscopists to crack proteins that are currently outside their grasp. Sanders, who until recently only had access to an $800-\mathrm{MHz}$ machine, says he has travelled to use a $900-\mathrm{MHz}$ spectrometer. "The same will be true for $1 \mathrm{GHz}$. People will travel for that."

But Jeremy Nicholson of Imperial College London says that grant-giving agencies may be losing interest in protein NMR spectroscopy. "It has been very successful in discovering the structures of a lot of very interesting, important proteins," he says. "But very few of those discoveries have translated into anything that has made a big mark in medicine."

Nicholson does foresee medical applications, however, particularly in metabolic profiling, which he helped to pioneer. Researchers generate complex

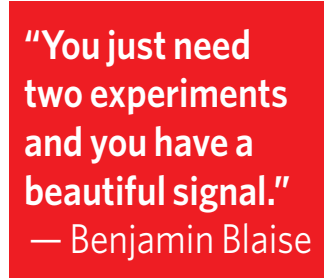

ies using samples from children with urinary problems. Blaise was one of the first people to use the new spectrometer. "It's amazing," he says. Formerly, he would run each of his samples about 100 times on the 900-MHz machine before getting a strong signal. Now, "you just need two experiments and you have a beautiful signal", he says.

\section{Watching reactions}

The metabolic profiling work also signals a return to NMR spectroscopy's roots in chemistry. Analytical chemists will be able to do similar kinds of chemical profiles for things such as water and soil samples to measure industrial effects. And high-field NMR spectroscopy could be used to analyse chemical reactions as they take place on the surface of large particles, such as chemical catalysts, which are often attached to grains of silica.

Here, the concentration of the active sample is often only around $1-2 \%$ of the total. The 1-GHz spectrometer "opens up new horizons", says chemist Christophe Copéret of the Lyon School of Chemistry Physics and Electronics. "We can study reactions that were not amenable to NMR at lower field."

There are still questions about whether the increase in field strength is worth the cost. Most NMR spectroscopy work doesn't require such large magnetic fields. Many protein structures can easily be deciphered at field strengths of less than $800 \mathrm{MHz}$, and $400 \mathrm{MHz}$ is usually enough to check the purity and structure of compounds in chemistry during organic synthesis.

The CRMN already had a 900$\mathrm{MHz}$ spectrometer before the 1-GHz machine arrived and it cost half as much. Given that for chemical 'fingerprints' of body fluids such as urine, plasma or tissue - or even whole organisms such as nematode worms - which are analysed to tease out the effects of drugs, disease or environmental changes on the metabolism. Higher field strength helps to differentiate the thousands of different metabolites.

Benjamin Blaise, a PhD student and trainee medic at the University of Lyon, is carrying out metabolic profiling studspectra improves in step with field strength, the extra $€ 6$ million seems steep for an improvement of around $10 \%$.

Emsley sees the machine as a resource for the entire research community. With the European Union contributing towards the running costs of the facility, up to 200 days a year will be allocated to users from other labs - many from outside France. Even relatively well- equipped labs would be lucky to have a 600 $\mathrm{MHz}$ machine. An increase of $400 \mathrm{MHz}$ is "an astonishing difference", Emsley says.

He points out that as magnetic field strength has increased in the past, new techniques have cropped up to exploit them. For example, TROSY came along just as NMR spectrometers with fields higher than $700 \mathrm{MHz}$ were becoming more widely available.

Sanders agrees. "In the past, every time there has been a big increase in the size of the magnet, NMR has leapt forward," he says. "Based on his tory, one might guess that there will be some breakthrough that results from this."

Kurt Wüthrich, who won the 2002 Nobel Prize in Chemistry for the development of NMR spectroscopy to determine protein structures, cautions that the step from $950 \mathrm{MHz}$ to $1 \mathrm{GHz}$ is small compared with the big hikes in field strength that have taken place since the early 1960s. For instance, fields had increased from around $60 \mathrm{MHz}$ to $600 \mathrm{MHz}$ by the mid-1980s. "There's now talk of 1.2 - and $1.3-\mathrm{GHz}$ machines," says Wüthrich, who is based at the Swiss Federal Institute of Technology in Zurich and the Scripps Research Institute, in La Jolla, California. "But in the past, relative increases were bigger."

The inroads that NMR spectroscopy has made into previously intractable problems hasn't just been driven by the increases in field strength. Other improvements in technology have contributed - such as the probe heads used to pulse samples with radio waves and measure resonances, and the computers that crunch the data. There have been big advances in sample preparation too, such as stereo-array isotope labelling (SAIL). In SAIL, proteins are made from specially labelled amino acids and these proteins produce much better spectra in NMR experiments.

Jim Emsley, Lyndon's father, says that the 1-GHz machine will provide a worthy challenge for his son. The technical advances of recent years and the funding secured by the Lyon centre are all signs that NMR spectroscopy remains a dynamic field. "As long as the science keeps saying 'Yes, it is worthwhile', that impetus will continue," he says. "So the onus now, obviously, is on Lyndon's laboratory."

Ananyo Bhattacharya is online news editor for Nature. 